\title{
Characterization of VdASP F2 Secretory Factor from Verticillium dahliae by a Fast and Easy Gene Knockout System
}

\author{
Chengjian Xie, ${ }^{1,2}$ Qiaoling $\mathrm{Li}^{3}{ }^{3}$ and Xingyong Yang ${ }^{1,2}$ \\ ${ }^{1}$ School of Life Sciences and; ${ }^{2}$ The Chongqing Key Laboratory of Molecular Biology of Plant Environmental Adaptations, \\ Chongqing Normal University, Chongqing 401331, China; and ${ }^{3}$ Chongqing Institute of Medicinal Plant Cultivation, Chongqing \\ 408435, China
}

Accepted 12 March 2017.

\begin{abstract}
The vascular wilt fungus Verticillium dahliae produces persistent resting structures known as microsclerotia, which enable long-term survival of this plant pathogen in soil. The completed genome sequence of $V$. dahliae has facilitated large-scale investigations of individual gene functions using gene-disruption strategies based on Agrobacterium tumefaciens-mediated transformation. However, the construction of gene-deletion vectors and screening of deletion mutants have remained challenging in $V$. dahliae. In this study, we developed a fast and easy gene knockout system for $\boldsymbol{V}$. dahliae using ligation-independent cloning and fluorescent screening. We identified secretory factor VdASP $F 2$ in a T-DNA insertion library of $V$. dahliae and deleted the $V d A S P F 2$ gene using the developed knockout system. Phenotypic analysis suggests that $V d A S P F 2$ is not necessary for $V$. dahliae growth on potato dextrose agar under various stress conditions. However, on semisynthetic medium or under limited nutrient conditions at lower temperatures, the VdASP F2 deletion mutant exhibited vigorous mycelium growth, less branching, and a significant delay in melanized microsclerotial formation. Further assessment revealed that $V d A S P F 2$ was required for the expression of $V D H 1$ and $V M K 1$, two genes involved in microsclerotial formation. Cotton inoculated with the VdASP F2 deletion mutant wilted, demonstrating that VdASP F2 is not associated with pathogenicity under normal conditions. However, after inducing microsclerotial formation and incubation at low temperatures, cotton infected with the VdASP F2 deletion mutant did not exhibit wilt symptoms. In conclusion, our results show that $\operatorname{VdASP} F 2$ plays an important role in the response of $V$. dahliae to adverse environmental conditions and is involved in a transition to a dormant form for prolonged survival.
\end{abstract}

Verticillium dahliae is a widespread and destructive soilborne fungus that causes wilt disease in hundreds of dicotyledonous plant species, including herbaceous annuals, perennials, and woody plants (Fradin and Thomma 2006; Klosterman et al. 2009). This leads to severe economic losses, especially in cotton production (James 2002). V. dahliae is particularly difficult to control because the fungus produces melanized structures known as

Corresponding author: X. Yang; Telephone: +86-13452957948;

Fax: +86-02365910315; E-mail: yangxy94@swu.edu.cn

*The $\boldsymbol{e}$-Xtra logo stands for "electronic extra" and indicates that one supplementary table and four supplementary figures are published online.

(c) 2017 The American Phytopathological Society microsclerotia (Rauyaree et al. 2005). These microsclerotia are dormant structures that allow the fungus to exist in the soil without a host for many years (Fradin and Thomma 2006). After stimulation by root exudates, microsclerotia germinate and subsequently enter into the root to initiate new infection cycles; thus, they are the primary source of $V$. dahliae infection (Fradin and Thomma 2006). The molecular mechanisms involved in Verticillium wilt have been gradually uncovered with the discovery of genes involved in virulence, growth, conidiation, and development. VMK1 encodes a mitogen-activated protein kinase that is involved in signal transduction pathways that control cellular and developmental processes in response to environmental cues. This protein has been reported to be required for microsclerotial formation and pathogenicity in V. dahliae (Rauyaree et al. 2005). VDH1, a hydrophobin gene, is also involved in microsclerotial development but is not required for pathogenicity of $V$. dahliae (Klimes and Dobinson 2006; Klimes et al. 2008). Glutamic acid-rich protein 1 plays an important role in sensing limited nutrient conditions in $V$. dahliae and is involved in microsclerotial formation and virulence (Gao et al. 2010). Microsclerotial development also involves mitogen-activated protein kinase VdHog1 (Wang et al. 2016b), MADS-box transcription factor VdMcm1 (Xiong et al. 2016), and calcineurin-responsive zincfinger transcription factor Crz1 (Xiong et al. 2015). Several additional proteins involved in virulence have been identified, including cAMP-dependent protein kinase A (VdPkac1) (Tzima et al. 2010), G-protein $\beta$ subunit (Tzima et al. 2012), necrosis- and ethyleneinducing peptide-1-like proteins (Santhanam et al. 2013; Zhou et al. 2012), the transcriptional regulator VdSge1 (Santhanam and Thomma 2013), and Ave, an effector recognized by the tomato Ve1 immune receptor, which mediates tomato resistance against $V$. dahliae race 1 (de Jonge et al. 2012).

With the completion of the genome sequence of $V$. dahliae, large-scale gene functional analysis has become possible. Agrobacterium tumefaciens-mediated transformation (ATMT) is a convenient and effective method for transformation of V. dahliae conidia (Maruthachalam et al. 2011). However, the generation of gene-replacement constructs and identification of null mutants from a large number of transformants remain limiting factors. To construct gene-replacement vectors, enzyme digestion or fusion polymerase chain reaction (PCR) are conventionally used (Szewczyk et al. 2007); however, these are usually time consuming and, sometimes, appropriate doublerestriction enzyme digestion sites cannot be found. Several convenient cloning techniques have been introduced to facilitate vector construction, such as the one-step construction of Agrobacterium recombination-ready plasmids method (Paz et al. 2011), the USER cloning technique (Wang et al. 2016a), 
and Saccharomyces cerevisiae-based recombination methods (Lu et al. 2014). Some methods have been employed to remove ectopic transformants. The herpes simplex virus thymidine kinase (HSVtk) is capable of converting 5-fluoro- $2^{\prime}$-deoxyuridine to a compound that is toxic to fungi; therefore, some researchers weed out ectopic insertions using HSVtk as a negative selectable marker (Ma et al. 2009; Wang et al. 2016a). In addition, KU70/KU80 (mus-51/mus-52) mutations have been used to construct fungal gene knockout background strains that exhibit a higher frequency of homologous recombination (Qi et al. 2015; Takahashi et al. 2006).

To explore the mechanism of microsclerotial development, we screened the $V$. dahliae T-DNA insertion transformant library and identified a mutant with less melanized microsclerotial formation. Using hiTAIL-PCR (Liu and Chen 2007), the T-DNA was identified to be inserted in a gene encoding allergen Asp F2-like proteins (VDAG_04551). These proteins are highly conserved sequences among fungi with signal peptides and are considered cell-wall components (He et al. 2015; Sentandreu et al. 1998). He et al. (2015) suggest that the disruption of the cerevisin gene affects microsclerotial formation and the virulence of $V$. dahliae, and VdASP F2 expression is significantly reduced in the cerevisin mutant (He et al. 2015). Asp F2 is a major allergen in Aspergillus fumigatus (Banerjee et al. 1996, 1998). Pra1, a pH-regulated Asp F2-like protein in Candida albicans, is a surface-associated and secreted Asp F2-like protein. Pra1 may play a role in cell morphogenesis by gene disruption (Sentandreu et al. 1998), inhibiting the activation of innate immune cells and neutrophil responses (Losse et al. 2011; Luo et al. 2010; Soloviev et al. 2007). However, the function of Asp F2 in plant-pathogenic fungi is poorly understood. In this study, we established a fast and easy gene knockout system for $V$. dahliae, which we used to create a V. dahliae V991 deletion mutant lacking VdASP F2 in order to determine its role in $V$. dahliae.

\section{RESULTS}

\section{Gene-replacement constructs.}

Exonuclease III (ExoIII) catalyzes the stepwise removal of mononucleotides from the 3'-hydroxyl termini of doublestranded DNA to generate long, compatible, cohesive ends between DNA inserts and cloning vectors. The annealed DNA complex is stable, and the ligation reaction can be omitted. The annealed DNA complex is transformed into Escherichia coli, and the noncomplementary regions (gaps and overhangs) are repaired by the bacteria. This technique has come to be known as ligation-independent cloning (LIC) (Liu et al. 2012).

PCR primers were designed to amplify two homologous arms of the VdASP F2 gene with 15 bp of the sequence overlapping the ends of the digested pGFP vector, which was constructed based on the pPK2 and constitutively expresses enhanced green fluorescent protein gene (eGFP) (Fig. 1A) (P1 and P4) or the hygromycin $\mathrm{B}$ phosphotransferase resistance gene cassette $\left(H_{y}{ }^{r}\right)$ (P2 and P3). The digested pGFP vector, Hyg ${ }^{r}$ cassette, and two homologous VdASP F2 arms were mixed with ExoIII (Fig. 1A) to generate long, compatible, cohesive ends between the pGFP vector, $\mathrm{Hyg}^{r}$ cassette, and VdASP F2 arms. The longer cohesive ends were able to anneal to each other and were more stable and, therefore, the ligation reaction could be omitted. Finally, the bacterial repair system resolved the overhangs or gaps in a predictable fashion to generate excisable inserts (Fig. 1A) (Li and Evans 1997). The gene-replacement construct was named pGFPVdASP F2, and approximately 15 to 30 colonies grew on the medium each time, all of which were correctly constructed vectors.

The gene disruption cassette was introduced into the host genome, where it was integrated by either homologous recombination or by ectopic recombination. Even if the target gene has been deleted in some transformants, extra ectopic integration of T-DNA is possible. Therefore, null mutants, ectopic insertions, and null mutants with ectopic insertions are all possible after Agrobacterium tumefaciens transformation (Fig. 1B).

\section{Gene deletion and complementation of $V d A S P F 2$ in $V$. dahliae.}

To verify gene-replacement constructs and analyze the function of VdASP F2 in V. dahliae, we constructed a gene knockout vector of VdASP F2 using the developed system. The knockout vector was transformed into $V$. dahliae via A. tumefaciens AGL1. As a negative selection marker, eGFP virtually eliminates all ectopic insertions in the knockout system. Green fluorescent screening was carried out to identify $V d A S P F 2$ null mutants (Fig. 2A). To confirm that VdASP F2 had been knocked out, primers were used to amplify the genome sequence at the insertion site (Fig. 1A), which returned a longer fragment if $V d A S P$ $F 2$ had been knocked out (Fig. 2B).

We obtained candidate null mutants based on the results of green fluorescent screening and PCR amplification (Fig. 2A and $\mathrm{B})$. For further confirmation, null mutants, ectopic insertions, and null mutants with ectopic insertions were analyzed by Southern blotting. The expected 2.6-kb band was observed on the Southern blot after $\mathrm{XbaI}$ digestion in null mutants, whereas multiple bands were observed in ectopic insertion mutants and null mutants with ectopic insertions (Fig. 2C). Reverse-transcription (RT)-PCR was performed to confirm the absence of VdASP F2 mRNA in VdASP F2 deletion strains (Fig. 2D). The results clearly indicate that the developed knockout system was successfully used to create a $V$. dahliae gene deletion mutant.

\section{VdASP F2 is a secretory protein}

\section{that is highly conserved among fungi.}

The VdASP F2 gene was present as a single copy in this $V$. dahliae strain. It has an open reading frame of 894 nucleotides with no introns and encodes a protein of 297 amino acids. BLAST searches against the National Center for Biotechnology Information GenBank database were performed to identify orthologs of VdASP F2 in order to examine the conservation of allergen Asp F2-like proteins in other organisms. Amino acid sequence analysis revealed that the resulting VdASP F2 orthologs were highly homologous, and the amino acid sequence was highly conserved among fungi. Alignments against orthologs from $S$. cerevisiae, Aspergillus fumigatus, and a few typical plant pathogens (Fusarium oxysporum and Sclerotinia sclerotiorum) are shown in Figure 3A.

Bioinformatics analysis revealed that Asp F2-like proteins contain a predicted N-terminal signal peptide. The signal peptide domain of VdASP F2 is located in the region between amino acid positions 1 and 18, suggesting that VdASP F2 may be a secreted protein (Fig. 3A). To confirm this hypothesis, eGFP and eGFP-tagged VdASP F2 (VdASP F2:eGFP) constitutive expression strains were constructed. Intense green fluorescence was detected in eGFP strains under fluorescence microscopy but only faint green fluorescence was observed in VdASP F2:eGFP strains (Fig. 3B). Strains were further inoculated in liquid Czapek-Dox medium and cultured with shaking. Western blotting revealed that VdASP F2:eGFP could be detected in the supernatant of the culture medium, indicating that VdASP F2 is indeed a secreted protein (Fig. 3C).

To analyze whether VdASP F2 is localized in plant tissue, we also expressed and purified fusion VdASP F2:eGFP without signal peptide ( $\triangle$ SP VdASP F2:eGFP-6His) (Supplementary Fig. S1A and B) because the signal peptide was cleaved off after the VdASP F2 was secreted by the $V$. dahliae strain. The locations of $\triangle$ SP VdASP F2:eGFP-6His and eGFP-6His are not significantly different in plant cells, as indicated by GFP 
fluorescence. In the in vitro root uptake assay, the purified proteins of both $\triangle$ SP VdASP F2:eGFP-6His and eGFP-6His were inoculated with cotton roots for $12 \mathrm{~h}$, and GFP fluorescence was observed on the root cells. However, the GFP fluorescence did not remain after washing twice (Supplementary Fig. S1C). Therefore, the results of the $\triangle$ SP VdASP F2:eGFP6 His uptake assay suggested that the secreted protein VdASP F2 cannot enter the plant cells.
Fungal growth and susceptibility to abiotic stress.

We constructed a geneticin (G418)-resistant vector to confirm that the phenotypic differences observed in the VdASP F2 deletion mutants were associated with the gene replacement event (Supplementary Fig. S2). A wild-type VdASP F2 gene under the control of its native promoter and terminator was introduced into the gene deletion mutant, and one G418-resistant transformant was selected for RT-PCR analysis of VdASP F2
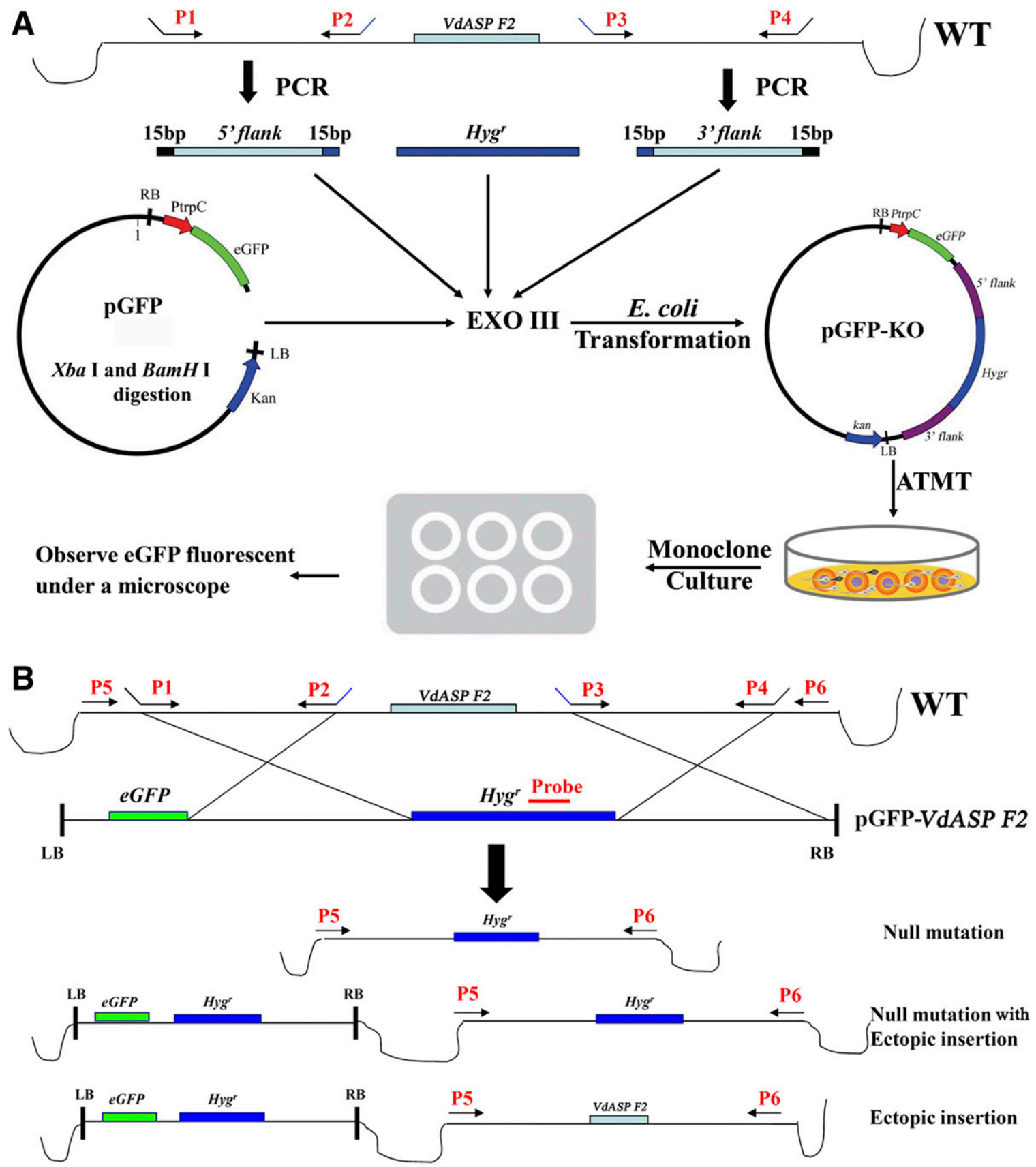

Fig. 1. Overview of fast and easy gene-knockout system in Verticillium dahliae. A, Two homologous arms of the target gene are amplified using polymerase chain reaction with primers containing 15 bp of sequence at the $5^{\prime}$ end and exonuclease III (ExoIII) digest to generate overhangs. P1 and P4 contain overhangs homologous to the pGFP vector, and P2 and P3 have overhangs homologous to the hygromycin B phosphotransferase resistance gene cassette ( Hyg $^{r}$ ), which allow for annealing. The annealing mixture is transformed into Escherichia coli, and gaps and overhangs are repaired by the bacteria to generate the circular knockout vector. The gene-deletion construct is then transformed into $\mathrm{V}$. dahliae via Agrobacterium tumefaciens-mediated transformation (ATMT). Transformants are screened for GFP fluorescence under a microscope. WT = wild type and eGFP = B, Homologous recombination creates three types of transformants: null mutants, ectopic insertion transformants, and null mutants with ectopic insertions. Null mutants do have not GFP fluorescence because the GFP gene was eliminated in the null mutants but ectopic transformants and null mutants with ectopic insertions exhibit green fluorescence. Primers P5 and P6 are used for identification of recombined DNA fragments, and a probe is used for Southern blotting. 
expression. This complemented mutant was then used in further phenotypic analyses for comparison with VdASP F2 knockout mutants. We assessed the vegetative growth and microsclerotial formation of the VdASP F2 deletion mutant, ectopic insertion transformant, complemented mutant, and wild-type strains under a variety of abiotic stresses. We found that vegetative growth and microsclerotial formation were not significantly different among the strains in the presence of $\mathrm{NaCl}, \mathrm{KCl}, \mathrm{H}_{2} \mathrm{O}_{2}$, high $\mathrm{pH}$, or sorbitol (Fig. 4A).

To investigate the role of VdASP F2 in $V$. dahliae growth during carbon uptake or utilization of specific carbon sources, the VdASP F2 deletion mutant, ectopic insertion transformant, complemented mutant, and wild-type strains were grown on Czapek-Dox medium with different carbon sources (glucose, sucrose, or cellulose). Analysis of colony diameters indicated no significant differences in radial growth rates among the different strains (Supplementary Fig. S3). All results indicated that VdASP F2 is not involved in carbon utilization or growth processes in $V$. dahliae. The VdASP F2 deletion strain, however, did exhibit less pigment and microsclerotial formation on carbon- or nitrogen-deficient medium and on semisynthetic (Czapek-Dox) medium (Fig. 4B).

\section{Disruption of VdASP F2 causes significantly delayed development of melanized microsclerotia in $\mathrm{V}$. dahliae.}

To explore the role of VdASP F2 in microsclerotial development, we grew the $V d A S P F 2$ deletion mutant, ectopic insertion transformant, complemented mutant, and wild-type strains on microsclerotia-inducing medium (MIM) to induce near-synchronous development of microsclerotia (Neumann and Dobinson 2003). After 15 days of growth, melanized microsclerotial production was observed in the ectopic insertion transformant, complemented mutant, and wild-type strains but not in the VdASP F2 deletion mutant. Melanized microsclerotial formation was observed in the VdASP F2 deletion mutant after 60 days (Fig. 5A). These results suggest that the VdASP F2 deletion delayed microsclerotial formation and development rather than eliminating it. The results are also consistent with growth on Czapek-Dox medium, where the VdASP F2 deletion mutant exhibited less production of microsclerotia in response
A

A

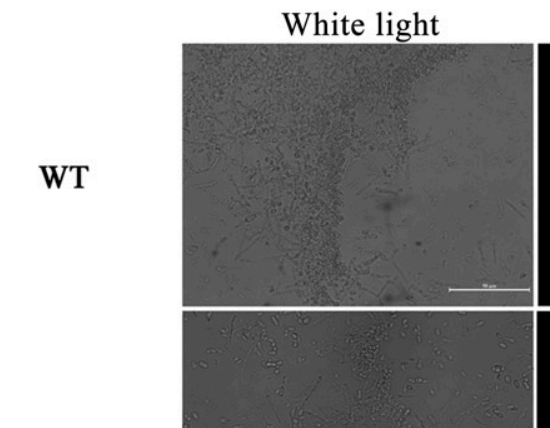

Null mutation
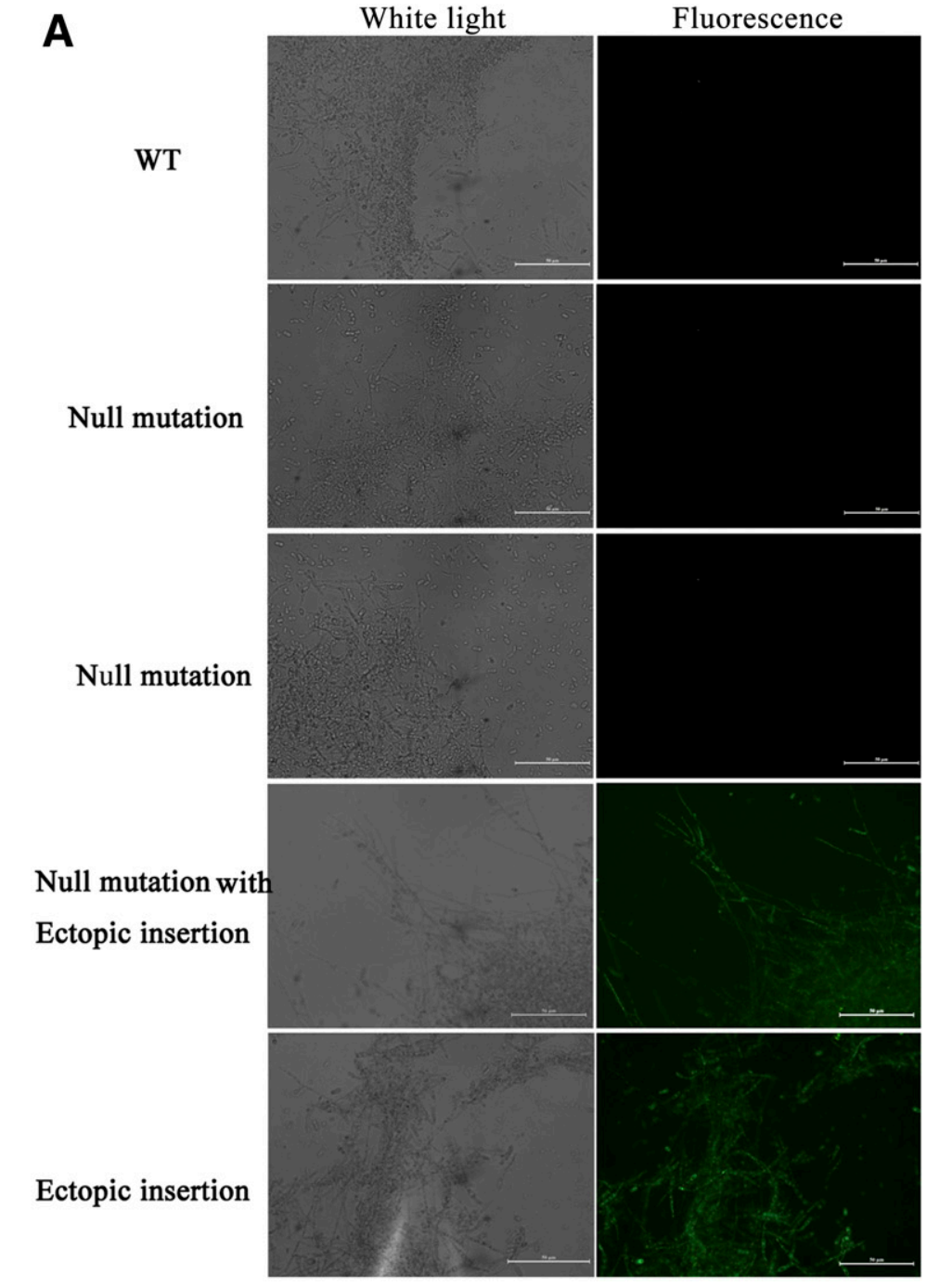

B

C
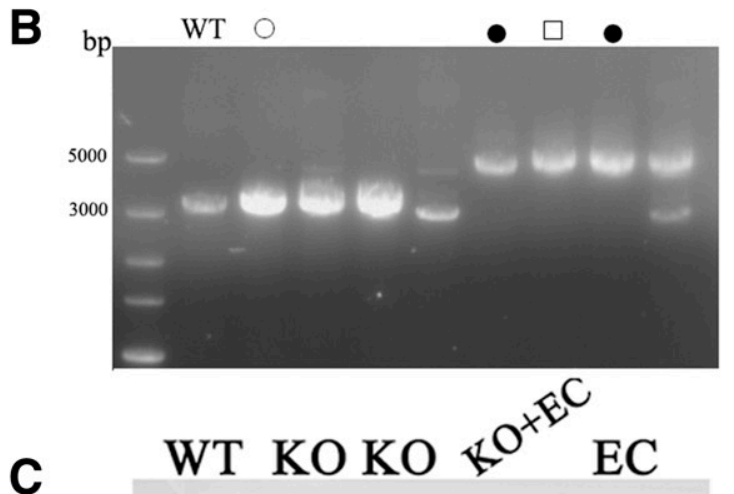

D

VDASP F2

$\beta$-tubulin

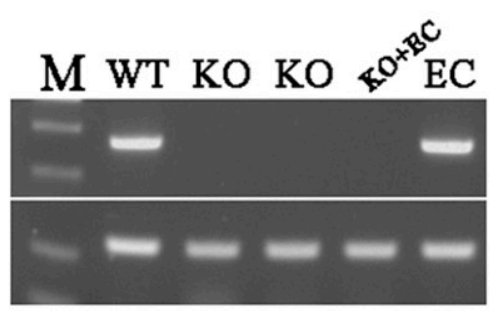

Fig. 2. Green fluorescent protein (GFP) screening and verification of VdASP F2 deletion mutants in Verticillium dahliae. A, GFP is not present in wild-type (WT) or null mutants but is present in ectopic insertion transformants and null mutants with ectopic insertions. B, A pair of primers complementary to the region outside of the two homologous arms of the VdASP F2 gene-deletion cassettes was used to verify the gene-deletion event. Open circles, closed circles, and open squares are used to represent ectopic insertion mutants (EC), null mutants (KO), and null mutants with ectopic insertions (KO+EC), respectively. C, Southern blotting indicates a single-copy insertion of the hygromycin B phosphotransferase resistance gene cassette $\left(\mathrm{Hyg}^{r}\right)$ in null mutants. Additional bands in the other lanes suggest ectopic insertions in ectopic insertion transformants and null mutants with ectopic insertions. D, reverse-transcription polymerase chain reaction reveals the elimination of the VdASP F2 gene in gene-deletion mutants. $\beta$-Tubulin was used as a reference gene. 
to nutrient limitation. Moreover, $V D H 1$ and, particularly, $V M K 1$, two genes that are involved in microsclerotial formation (Klimes and Dobinson 2006; Klimes et al. 2008; Rauyaree et al. 2005), exhibited lower expression in the VdASP F2 deletion mutant than in the wild type on MIM (Fig. 5B). These results demonstrate that VdASP F2 is related to microsclerotial formation.

Finally, we examined the mycelial growth and melanized microsclerotial formation of the VdASP F2 deletion mutant under extremely limited nutrient conditions by analyzing microsclerotial morphology in water agar medium (López-Escudero et al. 2012). Mycelial growth was extremely slow in the ectopic transformant, complemented mutant, and wild-type strains but the VdASP F2 deletion mutant exhibited vigorous aerial mycelium growth (Fig. 5C). In addition, the mycelia of the VdASP $F 2$ deletion mutant formed fewer branching hyphae than those of other strains (Fig. 5C). In particular, some substrate mycelium aggregates containing a dark, melanized outer layer formed in the ectopic transformant, complemented mutant, and wild-type strains but not in the VdASP F2 deletion mutant
(Fig. 5C and D). These hyphal aggregates eventually developed into microsclerotia.

We also examined the mycelial growth and melanized microsclerotial production of the VdASP $F 2$ deletion mutant under low temperature. None of the $V$. dahliae strains exhibited microsclerotial formation at $26^{\circ} \mathrm{C}$; however, except for the $V d A S P F 2$ deletion mutant, they formed microsclerotia more quickly when inoculated at $8^{\circ} \mathrm{C}$. The $\operatorname{VdASP} F 2$ deletion strain always exhibited vigorous mycelial growth on potato dextrose agar (PDA) with nitrocellulose membranes (Fig. 5E and F). These results suggest that VdASP F2 is involved in the response of $V$. dahliae to environmental conditions such as nutritional deficiency or low temperature but it is not necessary for microsclerotial formation under nutrient-rich conditions.

\section{VdASP F2 is not required for pathogenicity but is necessary for survival} of $V$. dahliae under adverse circumstances.

We subsequently assessed the role of VdASP F2 in the pathogenicity of $V$. dahliae by inoculating 3-week-old upland

A
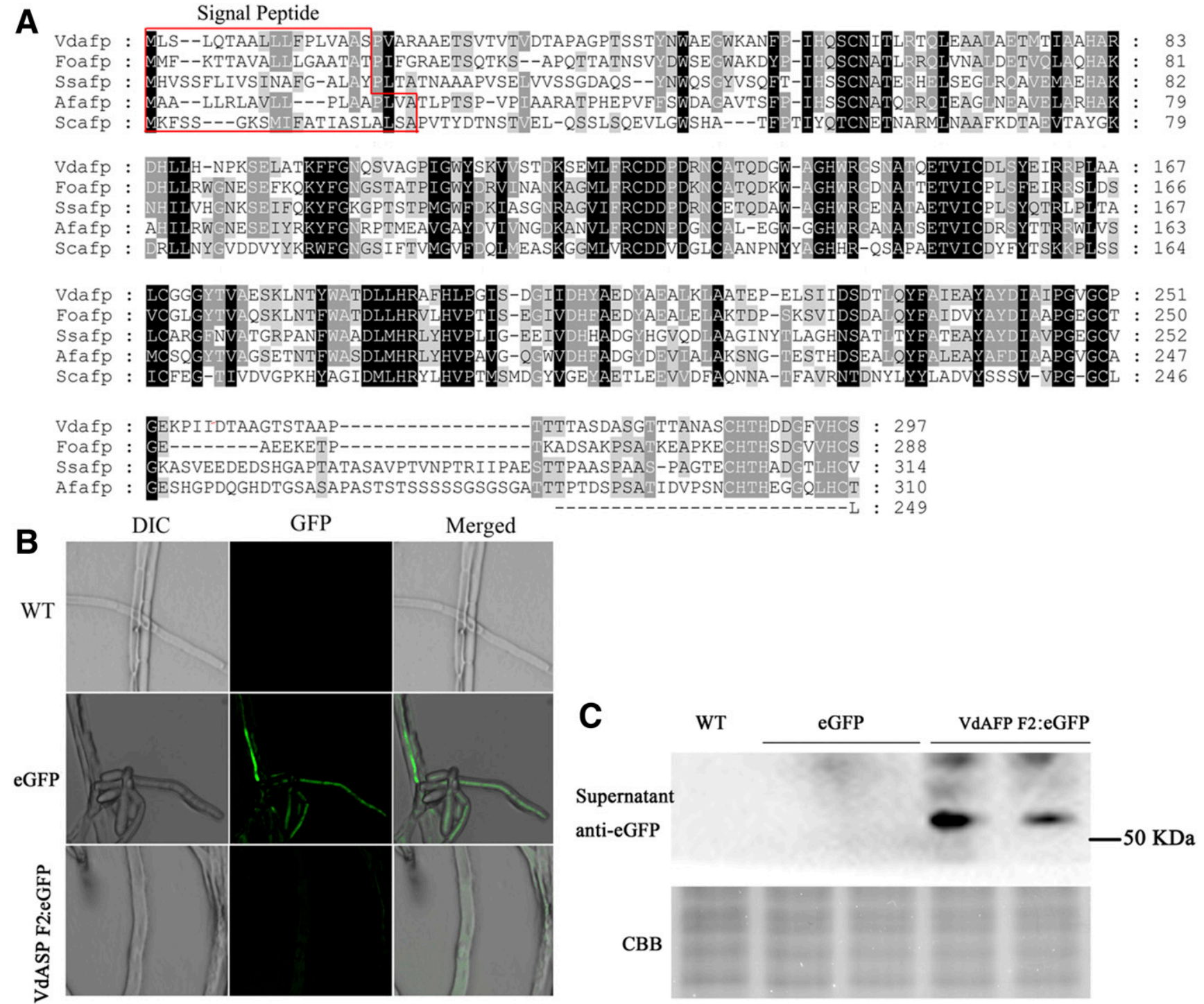

Fig. 3. VdASP F2 is a secreted protein in Verticillium dahliae. A, Multiple alignments indicate that ASP F2 is highly conserved among fungi. Protein sequences from top to bottom are derived from V. dahliae, Fusarium oxysporum, Saccharomyces cerevisiae, Sclerotinia sclerotiorum, and Aspergillus fumigates. B, Under differential interference contrast (DIC) and fluorescence (UV) microscopy, intense green fluorescence was detected in constitutively expressed eGFP strains, while only faint green fluorescence was observed in VdASP F2:eGFP strains. WT = wild type. C, Western blot analysis with total proteins isolated from liquid Czapek-Dox medium. Bands (58 kDa) were detected in VdASP F2:eGFP strains but not in WT or eGFP strains. Coomassie brilliant blue staining reveals equal loading of proteins used for Western blot analysis. 
cotton seeds (Gossypium hirsutum 'Xinluzao-13') with the VdASP $F 2$ deletion mutant, ectopic insertion transformant, complemented mutant, and wild-type strains using root-dip inoculation. All four strains showed wilting symptoms 20 days after $V$. dahliae inoculation (Fig. 6A). Thus, VdASP F2 does not appear to be required for pathogenicity against $G$. hirsutum.

$V$. dahliae is able to survive for 10 to 15 years in soil owing to the formation of microsclerotia (Fradin and Thomma 2006). To form microsclerotia and mimic V. dahliae overwintering in soil, we mixed the four $V$. dahliae strains with soil and incubated them at $8^{\circ} \mathrm{C}$ for 1 week to form microsclerotia. We then incubated them for 2 months at $-20^{\circ} \mathrm{C}$ to simulate winter conditions, and 6-week-old G. hirsutum seedlings were transferred into soil with these strains. Cotton plants infected with the ectopic transformant, complemented mutant, and wild-type strains exhibited chlorosis, wilting, and necrosis symptoms. However, those infected with the VdASP F2 deletion mutant did not (Fig. 6B).

In order to analyze the correlation between pathogen growth within plant tissues and symptom severity, quantitative PCR analysis was used to analyze the level of $V$. dahliae DNA in the aboveground stem of cotton. We seldom detected $V$. dahliae DNA in G. hirsutum infected by the low-temperature pretreated VdASP F2 deletion mutant (Fig. 6C). We speculate that the low infection rate of $G$. hirsutum is the result of low survival of the V. dahliae VdASP F2 mutant in the soil, due to its impaired ability to form microsclerotia.

\section{DISCUSSION}

Since the sequencing of the genome of $V$. dahliae, there has been a surge in investigations into the gene functions of $V$. dahliae using high-throughput gene deletion techniques.
ATMT is an effective method for the transformation of $V$. dahliae. However, the construction of gene-deletion vectors and screening of gene-deletion mutants limit the efficiency of ATMT. LIC offers an alternative to restriction enzyme or ligase cloning. Although the use of T4 DNA polymerase and ExoIII provides an efficient technique for cloning ( $\mathrm{Li}$ and Elledge 2007; Liu et al. 2012), the resulting constructs are not stable, particularly when dealing with multifragment ligations. In addition, accurate deletion of endogenous genes remains challenging in $V$. dahliae because of nonhomologous end joining (Qi et al. 2015). In this study, we presented an approach for gene deletion in $V$. dahliae that is suitable for high-throughput manipulation, in which several time-consuming steps can be performed in a high-throughput manner. To increase the cloning efficiency of the gene-replacement construct, pGFP was digested by two 5' overhang cutters (XbaI and BamHI) rather than a blunt-end cutter (Liu et al. 2012), and a larger amount of the $\mathrm{Hyg}^{r}$ cassette was prepared through PCR rather than from the enzyme digestion vector (Liu et al. 2012). To eliminate the most ectopic insertion transformants, eGFP-fused nuclear localization signal (eGFP NLS), rather than eGFP, was used as a negative marker, making fluorescence easier to observe.

There is little information available regarding the function of VdASP F2 in plant-pathogenic fungi. To investigate the function of VdASP F2, we used a fast and easy gene-deletion system to delete VdASP F2 in $V$. dahliae. The VdASP F2 deletion mutant exhibited vigorous aerial mycelium growth and reduced the ability to produce microsclerotia at low temperatures and under limited nutrient conditions. However, the mutant could still form microsclerotia on the PDA medium. Microsclerotia are the primary long-term survival structures when $V$. dahliae
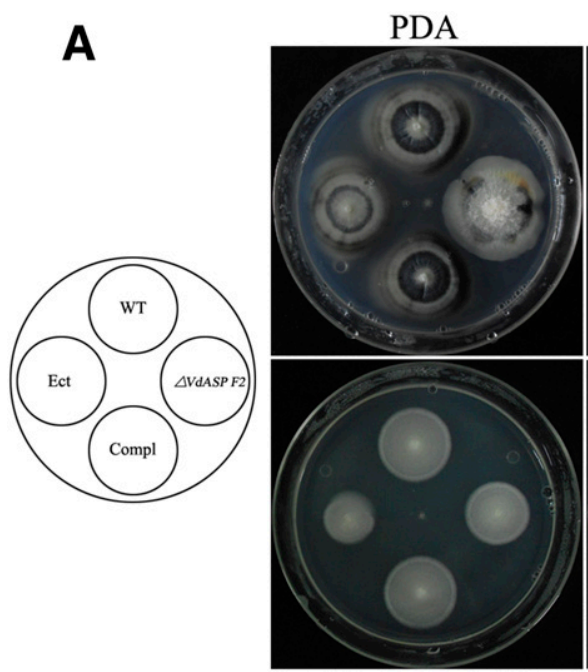

$\mathrm{H}_{2} \mathrm{O}_{2}$

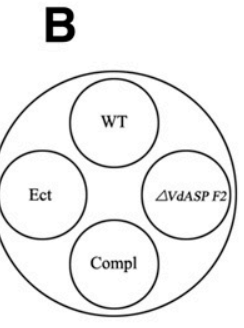

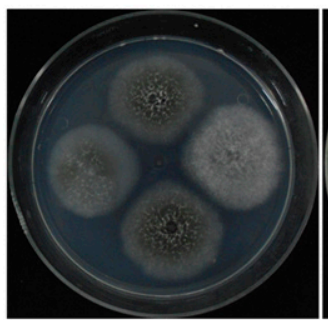

Czapek's medium

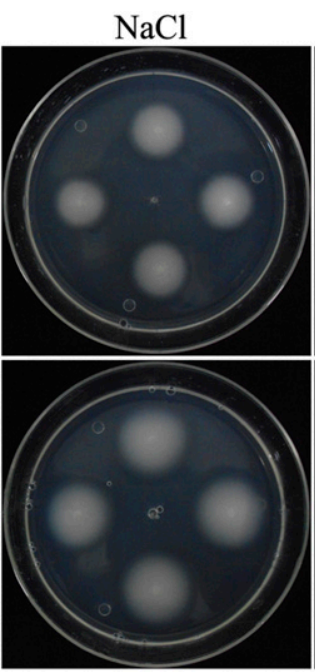

Sorbitol

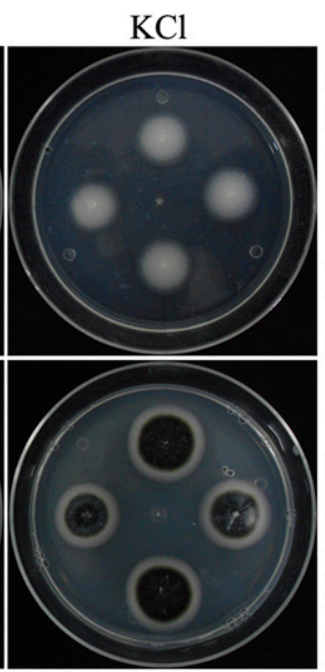

pH 11

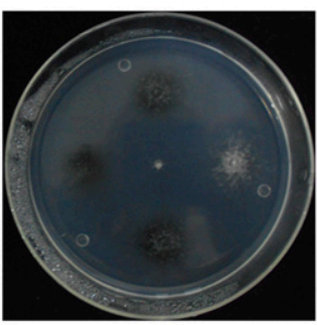

Nitrogen absent

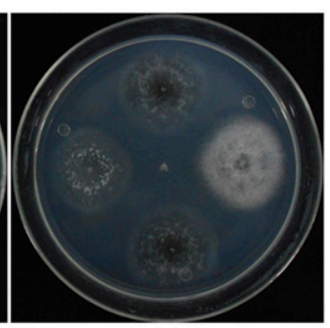

Carbon absent

Fig. 4. Susceptibility of VdASP F2 deletion mutants to abiotic stress. A, Growth of wild-type (WT) and mutant strains on potato dextrose agar (PDA) containing $0.5 \mathrm{M} \mathrm{NaCl}, 0.7 \mathrm{M} \mathrm{KCl}, 1.5 \mathrm{mM} \mathrm{H}_{2} \mathrm{O}_{2}$, or $1 \mathrm{M}$ sorbitol or grown at $\mathrm{pH} 11$. B, Growth comparisons among mutant and WT strains on Czapek-Dox medium without nitrogen or carbon source were conducted. $\triangle V d A S P F 2=V d A S P F 2$ deletion mutant, Compl = $\Delta V d A S P F 2$ mutant complemented with VdASP F2 WT allele, and Ect = ectopic transformant. 
encounters inhospitable environmental conditions. The results of the present study indicate that VdASP F2 cannot enter the plant cell and is considered a putative cell-wall-associated component (He et al. 2015), similar to the Asp F2-like protein Pra1, which was detected in the cell walls of C. albicans (Sentandreu et al. 1998).

We found that $V$. dahliae could form microsclerotia more quickly at 8 than at $26^{\circ} \mathrm{C}$. V. dahliae will survive if it can form microsclerotia at $8^{\circ} \mathrm{C}$ before it is transferred to $-20^{\circ} \mathrm{C}$. It is difficult for the $\operatorname{VdASP} F 2$ deletion mutant to survive at $-20^{\circ} \mathrm{C}$ because of the lack of microsclerotia. Liquid water turns into ice crystals below freezing temperatures and kills the cells. Thus, VdASP F2 is important for the quick formation of microsclerotia, helping $V$. dahliae survive exposure to poor environmental conditions. We mixed $V$. dahliae and soil and then subjected them to low temperatures, where it was difficult to maintain normal growth due to both limited soil nutrition and low temperature. In this case, the formation of microsclerotia is vital for $V$. dahliae survival. The VdASP F2 deletion mutant was impaired by its lack of microsclerotial protection. However, further research is required for a deeper understanding of the mechanism by which VdASP F2 regulates microsclerotial formation in the cell.

\section{MATERIALS AND METHODS}

\section{Strains and plants.}

V. dahliae strain V991, E. coli strain DH5a, and Agrobacterium tumefaciens strain AGL1 were used in this study, as was $G$. hirsutum L. Plants were grown in the greenhouse at $25^{\circ} \mathrm{C}$ under a daily cycle of $16 \mathrm{~h}$ of light and $8 \mathrm{~h}$ of darkness.

\section{Construction of binary and marker vectors.}

To construct a binary vector for pGFP, the 1-kb eGFP-NLS was amplified by PCR with PrimeSTAR HS DNA Polymerase (TaKaRa Dalian Biotechnology) and was ligated into the pSilent-1 vector between $X h o I$ and $K p n I$. The eGFP-NLS will highly express under the control of the $\operatorname{trp} C$ promoter (PtrpC). Using the pSilent-eGFP-NLS plasmid as a template, Ptrpc-eGFP-NLS was amplified and was ligated into the pPK2 vector between $\mathrm{XbaI}$ and EcoRI. The resulting plasmid was named pGFP. All primers in this study are listed in Supplementary Table S1.
A

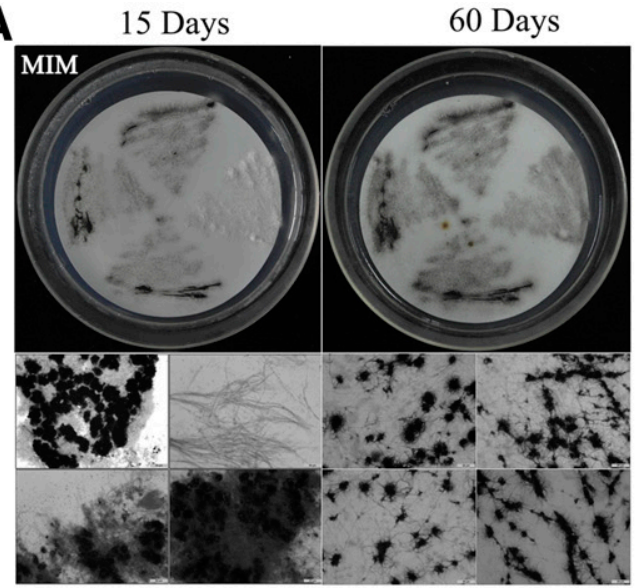

$\mathbf{E}$

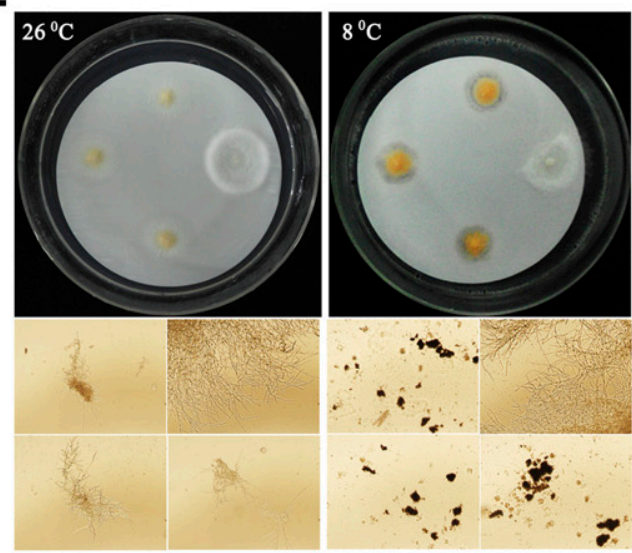

B

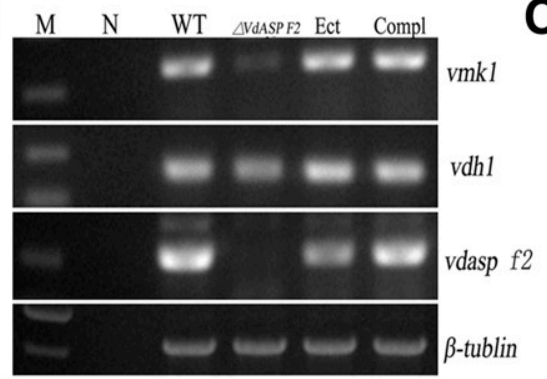

D

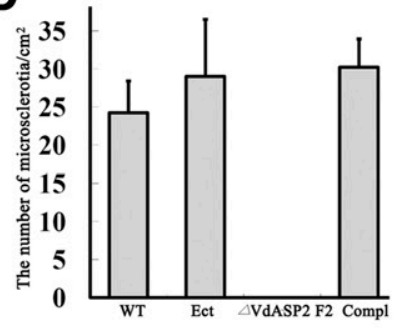

$\mathbf{F}$

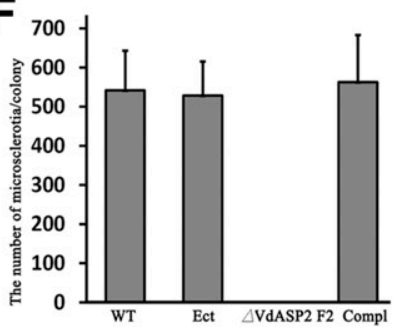

C

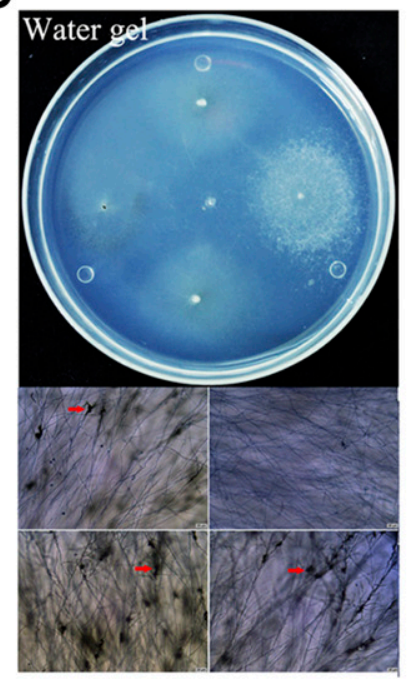

G

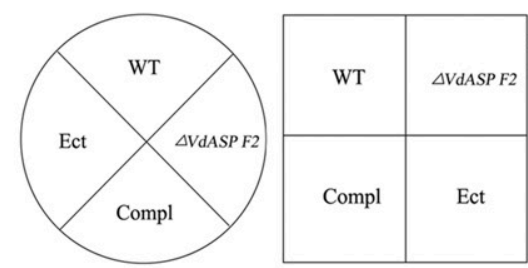

Fig. 5. Microsclerotial formation of VdASP F2 deletion mutant and pathogenicity analysis. A, Strains were cultured on solid microsclerotia-inducing medium (MIM) to induce microsclerotial formation. Mycelia and microsclerotia were scraped off the membrane for examination and counting under the microscope. The VdASP F2 deletion mutant exhibited denser mycelia with delayed and reduced microsclerotial development. Photos of the same Petri dishes were taken 15 days (left) and 60 days (right) postinoculation. B, Microsclerotial formation-related gene expression on MIM. Expression of mitogen-activated protein kinase (VMK1) and a hydrophobin gene $(V D H 1)$ were downregulated in the VdASP F2 deletion mutant. $\triangle V d A S P F 2=V d A S P F 2$ deletion mutant, Compl $=\Delta V d A S P$ $F 2$ mutant complemented with VdASP F2 WT allele, and Ect = ectopic transformant. Experiments were performed in triplicate. C, Colony, mycelial, and microsclerotial morphology. After 15 days of growth on water agar, the VdASP F2 deletion mutant exhibited vigorous aerial mycelia with reduced branching and did not form microsclerotia. Arrows indicate small microsclerotia. D, The histogram represents the number of microsclerotia from C. E, The microsclerotial formation was analyzed at both 26 and $8^{\circ} \mathrm{C}$. Pictures were taken 10 days after inoculation onto potato dextrose agar. F, The histogram represents the number of microsclerotia for strains inoculated at $8^{\circ} \mathrm{C}$. Experiments (D and F) were performed with nine replications, and the error bars represent standard deviations. G, Arrangement of all strains on the petri dish and microscope images. 
To produce the VdASP F2 gene-replacement vector, $\mathrm{Hyg}^{r}$ was amplified by PCR from pSilent-1. pGFP was digested with $5^{\prime}$ overhang cutters (XbaI and BamHI). The $\mathrm{Hyg}^{r}$ cassette, digested pGFP, and two homologous arms were purified by DNA Gel Extraction Kit (TaKaRa Dalian Biotechnology) and eluted using double-distilled $\mathrm{H}_{2} \mathrm{O}$ rather than elution buffer, because EDTA can inhibit ExoIII cleavage. The $\mathrm{Hyg}^{r}$ cassette and digested pGFP can be prepared and stored for future use. Two homologous arms, each comprising $1.5 \mathrm{~kb}$ from one side of the VdASP F2 open reading frame, were amplified using the $V$. dahliae genome database (www.broadinstitute.org). PrimeSTAR HS DNA Polymerase (TaKaRa Dalian Biotechnology) was used, attaining maximum fidelity and avoiding addition of $3^{\prime}$ A overhangs, which inhibit ExoIII cleavage. The underlined sequences in Supplementary Table S1 are reverse-complemented counterparts of the pGFP (P1 and P4) or $\mathrm{Hyg}^{r}$ cassette (P2 and $\mathrm{P} 3$ ). The pGFP vector (25 to $50 \mathrm{ng}$ ), Hyg $^{r}$ cassette (50 to $100 \mathrm{ng}$ ), two homologous VdASP F2 arms (25 to $50 \mathrm{ng}$ each), and $1 \mu \mathrm{l}$ of $10 \times$ ExoIII buffer were mixed in one reaction tube. The tube was prechilled on ice for $2 \mathrm{~min}$, and then $1 \mu \mathrm{l}$ of ExoIII (20 units) was added to the tube and gently mixed. After $90 \mathrm{~min}$ of incubation at $0^{\circ} \mathrm{C}, 1 \mu \mathrm{l}$ of $0.5 \mathrm{M}$ EDTA was added to stop the reaction. The enzyme was inactivated at $65^{\circ} \mathrm{C}$ for $5 \mathrm{~min}$. After cooling on ice, the mixture was transformed into $E$. coli component cells and cultured at $37^{\circ} \mathrm{C}$ overnight. Positive transformants were identified by colony PCR.

For the complementation assay, a 3.4-kb PCR product containing a $1.5-\mathrm{kb}$ upstream sequence, the full-length VdASP F2
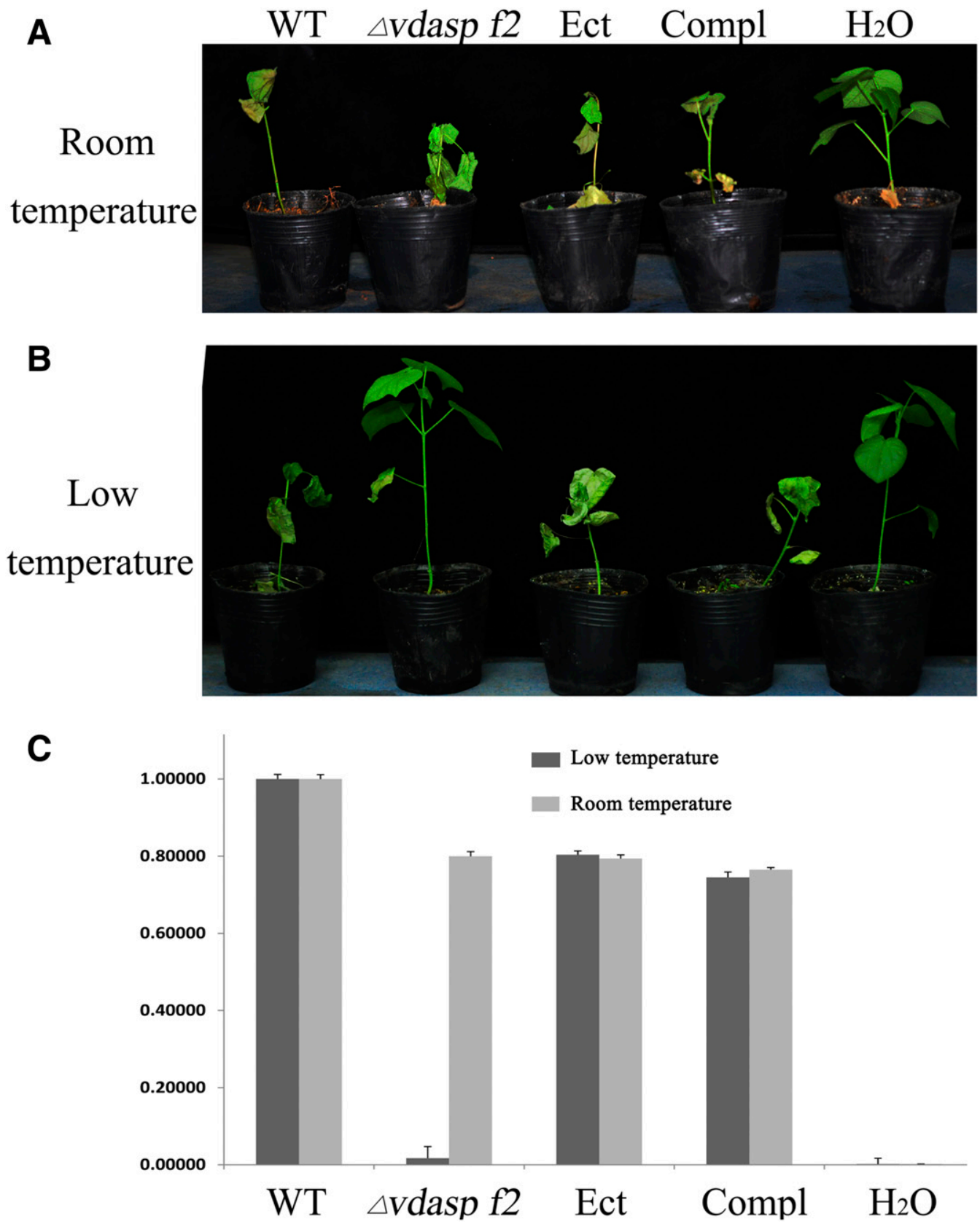

Fig. 6. Reduced virulence of the VdASP F2 mutant against Gossypium hirsutum. A, One-month-old G. hirsutum was planted in soil and kept at room temperature with conidia of wild-type (WT), $\triangle V d A S P F 2$ mutant, ectopic insertion mutant (Ect), and complementation (Compl) strains. B, G. hirsutum was planted in soil and kept at low temperature. Photographs were taken 20 days postinoculation. C, Verticillium dahliae DNA levels were estimated by quantitative polymerase chain reaction using total G. hirsutum DNA isolated from the aboveground stems of three plants per sample. The experiment was repeated three times, and the vertical bars indicate the standard errors. These results were automatically calculated by the CFX Manager Software (Bio-Rad). 
coding region, and a $1-\mathrm{kb}$ downstream sequence was amplified from $V$. dahliae V991 genomic DNA using primers compl F/R and cloned into the EcoRV/XbaI sites of the pG418 vector to generate pG418-VdASP F2, thereby conferring G418 resistance used for reintroduction of the wild-type $V d A S P F 2$ gene into the gene-deletion mutant. All gene-deletion vectors and genecomplementation vectors were transformed into A. tumefaciens strain AGL1 for subsequent $V$. dahliae transformation. The pG418 vector was constructed in our lab based on the pPK2 backbone.

To characterize the VdASP F2 protein, VdASP F2 was tagged with eGFP using fusion PCR. VdASP F2:eGFP and eGFP were first cut with SalI and KpnI and inserted into pSilent-1 (digested with $\mathrm{XhoI}$ and $K p n \mathrm{I}$ ); next, expression cassettes (PtrpC-VdASP F2:eGFP-TtrpC and PtrpC-eGFP-TtrpC) were amplified and cloned into the EcoRV/XbaI sites of the pG418 vector to generate the pG418-VdASP F2:eGFP and pG418eGFP vectors. These were then used for further characterization assays (see below).

In order to express proteins, VdASP F2-eGFP without signal peptide and eGFP was amplified by PCR with PrimeSTAR HS DNA Polymerase (TaKaRa Dalian Biotechnology) from VdASP F2:eGFP. $\triangle$ SPVdASP F2-eGFP-6His and eGFP-6His were digested by the restriction enzyme KpnI, followed by ligation with the binary vector PS1300, kindly provided by Dr. Jing Maofeng (Nanjing Agricultural University), which was digested with SmaI and KpnI.

\section{Fungal transformations.}

ATMT of $V$. dahliae was performed as described previously (Maruthachalam et al. 2011). pGFP-VdASP F2 transformants were selected on PDA supplemented with cefotaxime at $500 \mu \mathrm{g} / \mathrm{ml}$ and hygromycin B at $50 \mu \mathrm{g} / \mathrm{ml}$, whereas pG418VdASP F2 transformants were selected on PDA supplemented with cefotaxime at $500 \mu \mathrm{g} / \mathrm{ml}$ and G418 at $60 \mu \mathrm{g} / \mathrm{ml}$. To verify homologous recombination, the transformants were inoculated into six-well plates and then observed under fluorescence microscopy to exclude ectopic transformations. $V$. dahliae genomic DNA was extracted by the cetyltrimethylammonium bromide method, as described previously (Saghai-Maroof et al. 1984). The genomic DNA was used as a template for PCR amplification with primers P5 and P6 to confirm the targeted gene knockout. The deletion of VdASP F2 and its transcripts were further confirmed by Southern hybridization and RT-PCR. Complementation of VdASP F2 deletion mutants was also confirmed by Southern and RT-PCR.

\section{Bioinformatics analysis.}

VdASP F2 homologs from other fungi were identified by BLASTP. Sequences were aligned using MegAlign software (DNA STAR, Inc.). Signal peptide analysis was carried out using the signal peptide prediction program SignalP 4.1 Server (Petersen et al. 2011).

\section{Protein precipitation and Western blotting.}

To determine whether VdASP F2 was secreted into the liquid cultures, the VdASP F2:eGFP, eGFP, and wild-type strains were cultured in liquid Czapek-Dox medium at $25^{\circ} \mathrm{C}$ for 15 days at $180 \mathrm{rpm}$. The culture broth was centrifuged at $12,000 \times g$ for $15 \mathrm{~min}$ and filtered through three layers of Whatman filter paper to remove hyphal fragments. The volume of supernatant was reduced using a dialysis bag (MWCo $10 \mathrm{kDa}$; Sangon Biotech) and PEG 20,000, and then $30 \%$ (wt/vol) sucrose was added into the concentrated supernatant. The protein was extracted using an equal volume of $1 \mathrm{M}$ Tris-saturated phenol $(\mathrm{pH}$ 8.0). The mixture was homogenized and incubated on ice for $5 \mathrm{~min}$ before the upper phenol phase was collected and the proteins were precipitated with five volumes of $0.1 \mathrm{M}$ ammonium acetate in methanol overnight at $-20^{\circ} \mathrm{C}$. After centrifugation at $12,000 \times g$ for $15 \mathrm{~min}$, the protein pellets were washed once with methanol and $80 \%$ (vol $/ \mathrm{vol})$ acetone. The protein pellets were resuspended in protein loading buffer. Proteins were separated by sodium dodecyl sulfate polyacrylamide gel electrophoresis gel (12.5\%) before they were transferred onto a polyvinylidene diflouride membrane using a Western blotting apparatus (Bio-Rad). A monoclonal GFP antibody (Sino Biological Inc.) and a goat antimouse immunoglobulin $\mathrm{G}$ conjugated with HRP (OriGene) were used as primary and secondary antibodies, respectively. Blot signals were detected using Pierce ECL Western Blotting Substrate (Thermo Fisher Scientific).

\section{Fungal growth and susceptibility to stress conditions.}

The VdASP F2 deletion mutant, ectopic transformant, complemented mutant, and wild-type strains were grown on modified Czapek-Dox solid medium $\left(2 \% \mathrm{NaNO}_{3}, 0.1 \% \mathrm{~K}_{2} \mathrm{HPO}_{4}\right.$, $0.05 \% \mathrm{MgSO}_{4} \cdot 7 \mathrm{H}_{2} \mathrm{O}, 0.05 \% \mathrm{KCl}, 0.001 \% \mathrm{FeSO}_{4} \cdot 7 \mathrm{H}_{2} \mathrm{O}$, and $1.5 \%$ agar). The Czapek-Dox solid medium was supplemented with $1.5 \%$ glucose, $1.5 \%$ sucrose, or $0.5 \%$ cellulose as the sole carbon source. Mycelial growth was measured by colony diameter, and the hyphae and microsclerotia were visually estimated and compared.

Because microsclerotia play an important role in protecting $V$. dahliae from various types of stress, we evaluated the effects of $\mathrm{H}_{2} \mathrm{O}_{2}, \mathrm{pH}$, salt, sorbitol, and temperature stress on the vegetative growth of $V d A S P F 2$ deletion mutants inoculated on modified PDA.

\section{Microsclerotial production on microsclerotia-inducing or limited nutrient agar medium and RT-PCR.}

To determine whether VdASP F2 is required for microsclerotial production on MIM (Hu et al. 2013), the ectopic transformant, VdASP F2 knockout, complemented mutant, and wild-type strains were inoculated on MIM covered with a nitrocellulose membrane. After 15 or 60 days, hyphal and microsclerotial samples were taken for microscopic observation. To analyze whether the knockout of VdASP F2 influenced the expression of other genes related to microsclerotial formation, total RNA was extracted from hyphae and spores were collected from the nitrocellulose membrane covering the MIM. RNA integrity was confirmed by agarose gel electrophoresis. RT-PCR was performed with PrimeScrip RT Reagent Kit (TaKaRa Dalian Biotechnology). Expression of mitogen-activated protein kinase $(V M K 1)$ and a hydrophobin gene $(V D H 1)$ were analyzed by RT-PCR. PCR cycling consisted of an initial step of denaturation at $94^{\circ} \mathrm{C}$ for $2 \mathrm{~min}$, followed by 25 cycles of $94^{\circ} \mathrm{C}$ for $30 \mathrm{~s}, 57^{\circ} \mathrm{C}$ for $30 \mathrm{~s}$, and $72^{\circ} \mathrm{C}$ for $30 \mathrm{~s}$. All primers used in this study are listed in Supplementary Table S1).

The ectopic transformant, VdASP F2 deletion mutant, complemented mutant, and wild-type strains were inoculated on water agar to simulate limited nutrient conditions. Agar plugs were taken with a puncher for microscopic observation or counting. In order to view formation of microsclerotia under different temperatures, nitrocellulose membranes $(80 \mathrm{~mm}$ in diameter) were placed on PDA medium. Microsclerotia were scraped from membranes and counted using a hemocytometer. This experiment was repeated three times.

\section{Expression and purification of His-tagged proteins.}

For in planta expression, A. tumefaciens strain GV3101 with respective constructs was grown in Luria-Bertani media supplemented with appropriate antibiotics. For agroinfiltration assays, A. tumefaciens was resuspended in infiltration buffer

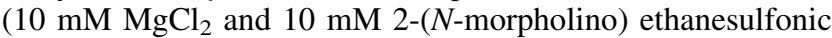
acid (MES), $\mathrm{pH}$ 5.6; and $100 \mu \mathrm{M}$ acetosyringone). The 
resuspended A. tumefaciens cells were diluted and mixed with P19 silencing suppressor in a 1:1 ratio at an optical density at $600 \mathrm{~nm}$ of 0.5 for each construct. Agroinfiltration experiments were performed on leaves of 6-week-old Nicotiana benthamiana plants.

Protein extraction was carried out according to the Lombardi et al. (2009) method, with minor modifications. N. benthamiana leaves were ground in liquid nitrogen in a precooled mortar and pestle. The samples were thawed in $3 \mathrm{ml}$ of cold extraction buffer (50 mM sodium phosphate [pH 8.0], $500 \mathrm{mM} \mathrm{NaCl}, 0.1 \%$ Nonidet P-40, 0.1\% $\beta$-mercaptoethanol, and $1 \mathrm{mM}$ phenylmethylsulfonyl fluoride) per gram of plant material. The samples were centrifuged at $12,000 \times g$ for $30 \mathrm{~min}$ at $4^{\circ} \mathrm{C}$ to pellet tissue debris. Imidazole was added to the supernatant for a final concentration of $10 \mathrm{mM}$. Then, $0.5 \mathrm{ml}$ of Ni-NTA agarose (Qiagen), preliminarily washed with extraction buffer, was added to the supernatant and incubated at $4^{\circ} \mathrm{C}$ on a rocker for $1 \mathrm{~h}$. Agarose was washed in $15 \mathrm{ml}$ of wash buffer (extraction buffer with $20 \mathrm{mM}$ imidazole). Bound His-tagged GFP was eluted with $250 \mathrm{mM}$ imidazole.

\section{In vitro cotton root uptake protein uptake.}

The experiment was carried out according to the $\mathrm{Gu}$ et al. (2011) method, with modifications. Cotton roots were washed with water thoroughly to remove any debris. Small root tips (approximately $1 \mathrm{~cm}$ ) were cut and placed into the protein solution (25 mM MES [pH 5.8] and protein at $1 \mathrm{mg} / \mathrm{ml})$ and incubated for $12 \mathrm{~h}$ at $28^{\circ} \mathrm{C}$. Half of the roots were washed twice in water for $2 \mathrm{~h}$ on an orbital shaker at $120 \mathrm{rpm}$. The roots were observed with an OLYMPUS DP70 microscope at excitations of 450 or $490 \mathrm{~nm}$.

\section{Infection assays.}

Six-week-old $G$. hirsutum seedlings were used in infection assays to evaluate the effect of VdASP F2 knockout on virulence using a root-dip inoculation method, as described previously (Santhanam and Thomma 2013). To prepare the inoculation, fungal cultures grown for 7 days in liquid potato dextrose broth medium were passed through several layers of cheesecloth to remove mycelia. Next, conidia $\left(10 \mathrm{ml} ; 1 \times 10^{7}\right)$ were mixed with the soil and keep at room temperature for 2 months. To mimic overwintering, $V$. dahliae spores were mixed with soil and kept at $8^{\circ} \mathrm{C}$ for 2 weeks to induce microsclerotial formation. They were then transferred to $-20^{\circ} \mathrm{C}$ for 2 months. Six-week-old G. hirsutum seedlings were planted in the soil with $V$. dahliae spores to assess pathogenicity. $G$. hirsutum was kept in an environmentally controlled growth room at $25 \pm 1^{\circ} \mathrm{C}$ and 60 to $70 \%$ relative humidity under a daily cycle of $12 \mathrm{~h}$ of light and $12 \mathrm{~h}$ of darkness. Pictures were taken 20 days after $V$. dahliae inoculation.

\section{V. dahliae biomass quantification in cotton.}

Aboveground stems of cotton were harvested and flash frozen in liquid nitrogen. The samples were ground to powder, approximately $1 \mathrm{~g}$ of which was used for DNA isolation. Quantitative RT-PCR was conducted by the CFX Connect Real-Time PCR Detection System (Bio-Rad) with SYBR Green Master Mix (TaKaRa Dalian Biotechnology). For $V$. dahliae biomass measurement, the primer pair Vd-F and Vd-R was designed for the internal transcribed spacer (ITS) 1 and ITS2 regions of the 5.8S ribosomal RNA gene of $V$. dahliae. The osmotic gene from G. hirsutum, amplified using the primer pair Gh-F and Gh-R, was used as an internal standard to normalize for differences in DNA template amounts. The average fungal biomass was determined using at least three $V$. dahliae-inoculated cotton samples. The experiments were repeated three times. PCR cycling started with an initial step of denaturation at $95^{\circ} \mathrm{C}$ for $3 \mathrm{~min}$, followed by 40 cycles of $95^{\circ} \mathrm{C}$ for $15 \mathrm{~s}, 55^{\circ} \mathrm{C}$ for $15 \mathrm{~s}$, and $72^{\circ} \mathrm{C}$ for $30 \mathrm{~s}$.

\section{ACKNOWLEDGMENTS}

This study was funded by the Chongqing Municipal Education Commission (grant number KJ1500324), Ph.D. Initial Funding from Chongqing Normal University (grant number 12XLB002), and the Key Foundation Project of Chongqing Normal University (grant number 2011XLZ09). The authors declare that they have no conflict of interest, and this article does not contain any studies with human participants or animals performed by any of the authors.

\section{LITERATURE CITED}

Banerjee, B., Greenberger, P. A., Fink, J. N., and Kurup, V. P. 1998 Immunological characterization of Asp $\mathrm{f} 2$, a major allergen from Aspergillus fumigatus associated with allergic bronchopulmonary aspergillosis. Infect. Immun. 66:5175-5182.

Banerjee, B., Kurup, V. P., Phadnis, S., Greenberger, P. A., and Fink, J. N. 1996. Molecular cloning and expression of a recombinant Aspergillus fumigatus protein Asp f II with significant immunoglobulin E reactivity in allergic bronchopulmonary aspergillosis. J. Lab. Clin. Med. 127: 253-262.

de Jonge, R., van Esse, H. P., Maruthachalam, K., Bolton, M. D., Santhanam, P., M. K., Saber, Zhang, Z., Usami, T., Lievens, B., Subbarao, K. V., and Thomma, B. P. H. J. 2012. Tomato immune receptor Ve1 recognizes effector of multiple fungal pathogens uncovered by genome and RNA sequencing. Proc. Natl. Acad. Sci. U.S.A. 109:5110-5115.

Fradin, E. F., and Thomma, B. P. 2006. Physiology and molecular aspects of Verticillium wilt diseases caused by V. dahliae and V. albo-atrum. Mol. Plant Pathol. 7:71-86.

Gao, F., Zhou, B.-J., Li, G.-Y., Jia, P.-S., Li, H., Zhao, Y.-L., Zhao, P., Xia, G.-X., and Guo, H.-S. 2010. A glutamic acid-rich protein identified in Verticillium dahliae from an insertional mutagenesis affects microsclerotial formation and pathogenicity. PLoS One 5:e15319.

Gu, B., Kale, S. D., Wang, Q., Wang, D., Pan, Q., Cao, H., Meng, Y., Kang, Z., Tyler, B. M., and Shan, W. 2011. Rust secreted protein Ps87 is conserved in diverse fungal pathogens and contains a RXLR-like motif sufficient for translocation into plant cells. PLoS One 6:e27217.

He, X. J., Li, X. L., and Li, Y. Z. 2015. Disruption of Cerevisin via Agrobacterium tumefaciens-mediated transformation affects microsclerotia formation and virulence of Verticillium dahliae. Plant Pathol. 64: 1157-1167

Hu, X., Bai, Y., Chen, T., Hu, D., Yang, J., and Xu, X. 2013. An optimized method for in vitro production of Verticillium dahliae microsclerotia. Eur. J. Plant Pathol. 136:225-229.

James C. 2002. Global review of commercialized transgenic Crops: 2001 Feature: Bt Cotton. ISAAA Briefs No. 26. The International Service for the Acquisition of Agri-biotech Applications (ISAAA), Ithaca, NY.

Klimes, A., Amyotte, S. G., Grant, S., Kang, S., and Dobinson, K. F. 2008 Microsclerotia development in Verticillium dahliae: Regulation and differential expression of the hydrophobin gene $V D H 1$. Fungal Genet. Biol. 45:1525-1532.

Klimes, A., and Dobinson, K. F. 2006. A hydrophobin gene, VDH1, is involved in microsclerotial development and spore viability in the plant pathogen Verticillium dahliae. Fungal Genet. Biol. 43:283-294.

Klosterman, S. J., Atallah, Z. K., Vallad, G. E., and Subbarao, K. V. 2009. Diversity, pathogenicity, and management of Verticillium species. Annu. Rev. Phytopathol. 47:39-62.

López-Escudero, F. J., Roca, J. M., Valverde-Corredor, A., and MercadoBlanco, J. 2012. Correlation between virulence and morphological characteristics of microsclerotia of Verticillium dahliae isolates infecting olive. J. Phytopathol. 160:431-433.

Li, C., and Evans, R. M. 1997. Ligation independent cloning irrespective of restriction site compatibility. Nucleic Acids Res. 25:4165-4166.

Li, M. Z., and Elledge, S. J. 2007. Harnessing homologous recombination in vitro to generate recombinant DNA via SLIC. Nat. Methods 4: 251-256.

Liu, Y., Li, S., Zhang, H., Wan, Z., Zhang, X., and Du, R. 2012. A one-step cloning method for the construction of somatic cell gene targeting vectors: Application to production of human knockout cell lines. BMC Biotechnol. 12:71.

Liu, Y. G., and Chen, Y. 2007. High-efficiency thermal asymmetric interlaced PCR for amplification of unknown flanking sequences. Biotechniques 43:649-656.

Lombardi, R., Circelli, P., Villani, M. E., Buriani, G., Nardi, L., Coppola, V., Bianco, L., Benvenuto, E., Donini, M., and Marusic, C. 2009. High-level HIV-1 Nef transient expression in Nicotiana benthamiana using the P19 gene silencing suppressor protein of Artichoke mottled crinckle virus. BMC Biotechnol. 9:96 
Losse, J., Svobodova, E., Heyken, A., Hube, B., Zipfel, P. F., and Jozsi, M. 2011. Role of $\mathrm{pH}$-regulated antigen 1 of Candida albicans in the fungal recognition and antifungal response of human neutrophils. Mol. Immunol. 48:2135-2143.

Lu, J., Cao, H., Zhang, L., Huang, P., and Lin, F. 2014. Systematic analysis of Zn2Cys6 transcription factors required for development and pathogenicity by high-throughput gene knockout in the rice blast fungus. PLoS Pathog. 10:e1004432.

Luo, S., Hartmann, A., Dahse, H. M., Skerka, C., and Zipfel, P. F. 2010. Secreted pH-regulated antigen 1 of Candida albicans blocks activation and conversion of complement C3. J. Immunol. 185:2164-2173.

Ma, J.-C., Zhou, Q., Zhou, Y.-H., Liao, X.-G., Zhang, Y.-J., Jin, D., and Pei, Y. 2009. The size and ratio of homologous sequence to non-homologous sequence in gene disruption cassette influences the gene targeting efficiency in Beauveria bassiana. Appl. Microbiol. Biotechnol. 82:891-898.

Maruthachalam, K., Klosterman, S. J., Kang, S., Hayes, R. J., and Subbarao, K. V. 2011. Identification of pathogenicity-related genes in the vascular wilt fungus Verticillium dahliae by Agrobacterium tumefaciens-mediated T-DNA insertional mutagenesis. Mol. Biotechnol. 49:209-221.

Neumann, M. J., and Dobinson, K. F. 2003. Sequence tag analysis of gene expression during pathogenic growth and microsclerotia development in the vascular wilt pathogen Verticillium dahliae. Fungal Genet. Biol. 38:54-62.

Paz, Z., Garcia-Pedrajas, M. D., Andrews, D. L., Klosterman, S. J., BaezaMontanez, L., and Gold, S. E. 2011. One step construction of Agrobacterium-Recombination-ready-plasmids (OSCAR), an efficient and robust tool for ATMT based gene deletion construction in fungi. Fungal Genet. Biol. 48:677-684.

Petersen, T. N., Brunak, S., von Heijne, G., and Nielsen, H. 2011. SignalP 4.0: Discriminating signal peptides from transmembrane regions. Nat. Methods 8:785-786.

Qi, X., Su, X., Guo, H., Qi, J., and Cheng, H. 2015. A ku70 null mutant improves gene targeting frequency in the fungal pathogen Verticillium dahliae. World J. Microbiol. Biotechnol. 31:1889-1897.

Rauyaree, P., Ospina-Giraldo, M. D., Kang, S., Bhat, R. G., Subbarao, K. V., Grant, S. J., and Dobinson, K. F. 2005. Mutations in VMK1, a mitogenactivated protein kinase gene, affect microsclerotia formation and pathogenicity in Verticillium dahliae. Curr. Genet. 48:109-116.

Saghai-Maroof, M. A., Soliman, K. M., Jorgensen, R. A., and Allard, R. W. 1984. Ribosomal DNA spacer-length polymorphisms in barley: Mendelian inheritance, chromosomal location, and population dynamics. Proc. Natl. Acad. Sci. U.S.A. 81:8014-8018.

Santhanam, P., and Thomma, B. P. H. J. 2013. Verticillium dahliae Sge1differentially regulates expression of candidate effector genes. Mol. Plant-Microbe Interact. 26:249-256.
Santhanam, P., van Esse, H. P., Albert, I., Faino, L., Nürnberger, T., and Thomma, B. P. H. J. 2013. Evidence for functional diversification within a fungal NEP1-like protein family. Mol. Plant-Microbe Interact. 26:278-286.

Sentandreu, M., Elorza, M. V., Sentandreu, R., and Fonzi, W. A. 1998. Cloning and characterization of PRA1, a gene encoding a novel $\mathrm{pH}$ regulated antigen of Candida albicans. J. Bacteriol. 180:282-289.

Soloviev, D. A., Fonzi, W. A., Sentandreu, R., Pluskota, E., Forsyth, C. B. Yadav, S., and Plow, E. F. 2007. Identification of pH-regulated antigen 1 released from Candida albicans as the major ligand for leukocyte integrin $\alpha \mathrm{M} \beta 2$. J. Immunol. 178:2038-2046.

Szewczyk, E., Nayak, T., Oakley, C. E., Edgerton, H., Xiong, Y., TaheriTalesh, N., Osmani, S. A., and Oakley, B. R. 2007. Fusion PCR and gene targeting in Aspergillus nidulans. Nat. Protoc. 1:3111-3120.

Takahashi, T., Masuda, T., and Koyama, Y. 2006. Enhanced gene targeting frequency in $k u 70$ and $k u 80$ disruption mutants of Aspergillus sojae and Aspergillus oryzae. Mol. Genet. Genomics 275:460-470.

Tzima, A., Paplomatas, E. J., Rauyaree, P., and Kang, S. 2010. Roles of the catalytic subunit of cAMP-dependent protein kinase A in virulence and development of the soilborne plant pathogen Verticillium dahliae. Fungal Genet. Biol. 47:406-415.

Tzima, A. K., Paplomatas, E. J., Tsitsigiannis, D. I., and Kang, S. 2012. The $\mathrm{G}$ protein $\beta$ subunit controls virulence and multiple growth- and development-related traits in Verticillium dahliae. Fungal Genet. Biol. 49:271-283.

Wang, S., Xing, H., Hua, C., Guo, H. S., and Zhang, J. 2016a. An improved single-step cloning strategy simplifies the Agrobacterium tumefaciensmediated transformation (ATMT)-based gene-disruption method for Verticillium dahliae. Phytopathology 106:645-652.

Wang, Y., Tian, L., Xiong, D., Klosterman, S. J., Xiao, S., and Tian, C. 2016b. The mitogen-activated protein kinase gene, VdHog1, regulates osmotic stress response, microsclerotia formation and virulence in Verticillium dahliae. Fungal Genet. Biol. 88:13-23.

Xiong, D., Wang, Y., Tang, C., Fang, Y., Zou, J., and Tian, C. 2015. VdCrz1 is involved in microsclerotia formation and required for full virulence in Verticillium dahliae. Fungal Genet. Biol. 82:201-212.

Xiong, D., Wang, Y., Tian, L., and Tian, C. 2016. MADS-Box transcription factor VdMcm1 regulates conidiation, microsclerotia formation, pathogenicity, and secondary metabolism of Verticillium dahliae. Front. Microbiol. 7:1192.

Zhou, B. J., Jia, P. S., Gao, F., and Guo, H. S. 2012. Molecular characterization and functional analysis of a necrosis- and ethylene-inducing, proteinencoding gene family from Verticillium dahliae. Mol. Plant-Microbe Interact. 25:964-975 